\title{
Can we reduce waste and waist together through EUPHORIA?
}

\author{
Veranika Lim, Fulya Yalvaç, Mathias Funk, Jun Hu and Matthias Rauterberg \\ Designed Intelligence Group, Department of Industrial Design \\ Eindhoven University of Technology, Den Dolech 2. 5612 AZ, The Netherlands \\ \{v.lim,f.yalvac,m.funk,j.hu,g.w.m.rauterberg\}@tue.nl
}

\begin{abstract}
Food waste has received little attention in pervasive computing. In this paper we discuss previous work which stresses the need for understanding how intelligent technology could prevent food waste in households. We present EUPHORIA, a community based social system that can track users' food waste potential and redirect behavior, through social influence strategies, towards more sustainable food related practices. To promote social interaction, the system recommends social recipes based on available food from different users to prevent waste as well as to provide a new pleasurable experience around food. A mobile application will be developed in three phases. In the first phase, the application allow users to log and track food waste related behavior patterns. Next, it will provide eco-feedback and social visibility of own and other users' food related behavior. Finally, a social recipe recommendation will be implemented. Findings are expected to provide a better understanding of consumers' social behavior around food waste.
\end{abstract}

\section{INTRODUCTION}

In recent years, the impact of food losses and food waste on food security has become a global concern. Previous estimates has indicated that one third to one half of the world's food becomes waste, while twice as much food is produced globally than is required by nutritional needs per living person [1] [2]. Still, food production will need to increase by 70 percent in order to feed 9 billion people by 2050 . Growing food that never reaches the consumers stomach seems to make 10 percent of rich countries' greenhouse gas emissions and waste 550 billion cubic meters of water globally [3]. With less than a quarter of wasted food, all the world's nearly one billion hungry people could be lifted out of malnourishment [4]. In response to this, FUSIONS (Food Use for Social Innovation by Optimizing Waste Prevention Strategies) was set up to work towards a 50 percent reduction of food waste generated in the EU and a 20 percent reduction in food chain resource inputs by 2020 [5]. UNEP [6] has set future goals for raising awareness on the value of food people eat, as well as the environmental impacts of their choices with the prospective to redirect consumption patterns to less resource-intensive foods. Hence, food waste prevention is the key approach to reduce environmental impacts of food related behavior in the next decennia.

To prevent food waste, actions on various levels are required, such as changes in policies, technological environment, education or individual consumption patterns etc. As final

This work is supported in part by the Erasmus Mundus Joint Doctorate in Interactive and Cognitive Environments (ICE), which is funded by the EACEA Agency of the European Commission under EMJD ICE FPA n 2010-0012. demand of food is located at the consumer level, consumers can be considered to be the most important drivers of overall resource consumption, waste generation and eventually the environmental impacts on our world [7]. In Europe and the US, roughly half of the total avoidable losses within the food chain is generated by households [1] [8] [9] [10]. Furthermore, a large study performed in the UK, illustrated that the avoidable food losses correspond to 21.3 percent of the purchases [11]. In 2008, in the US, 19 percent of the total amount of food supply was wasted at the consumer level [12]. Reducing food waste at the consumer level in Europe only is expected to have impacts on Gross Domestic Product (GDP) increase, land use within the EU and food security in Sub-Saharan Africa [13] [14].

Despite these findings, persuasive and pervasive sustainability research, has mainly put effort into areas such as sustainable energy consumption, resource consumption, and green transportation or are not tied to any specific topic [15]. Although ecological sustainability is an increasing concern in sustainability research, to date, food waste has received little attention. This has raised our interest in understanding how intelligent technology could improve resource efficiency and hence prevent food waste throughout consumers' every day lives. In addition, we aim at understanding how social influence strategies can be applied to food waste prevention. So far, the use and study of social media to motivate change is found to have promising potential but is under explored [16]. There is a need to study social behavior around food practices as it is a key aspect of daily life which could have enormous impacts on the design, use and hence the acceptance of technology. Therefore, we would like to draw on this opportunity for exploration.

The structure of this paper is as follows. In Section 2, we will present EUPHORIA, a project working towards a conceptual design of a social persuasive technology system. In Section 3, previous research on food related behavior will be discussed as well as current technological solutions for food waste prevention designed for consumers. In Section 4, we will provide our planned research approach. Finally in Section 5, conclusions, social implications and future research directions will be discussed.

\section{EUPHORIA}

EUPHORIA, which stands for Efficient food Use and food waste Prevention in Households through Increased Awareness, is a project aiming at designing a social system for the community that allows users to log and track their every day food 
related behavior and redirect these, through social influence, towards more sustainable food related practices promoting sustainable lifestyles. The main function of the system is to detect potential food waste and hence respond by providing recipes aiming at food waste prevention. Recipes will also be based on seasonal ingredients and healthy alternatives as much as possible. To promote social interaction and to gain more effective food waste prevention, the system will provide social recipes through a group based recipe recommendation system. This means that the system will combine available ingredients from different households that need to be consumed in time into one recipe. This social aspect is expected to provide a new pleasurable experience around food practices such as planning, purchasing, cooking and inter-cultural encounter.

To achieve this, we first aim at exploring and capturing variations in human behavior around food waste to identify opportunities for social computing. The mobile application can be used within a group of users and will be deployed and evaluated in three phases. In the first phase we will monitor and model food consumption and food waste behavior. Here, we aim at quantifying and understanding consumers' food intake and food waste flows related to context (e.g. time, temperature, type of day etc.) in order to specify determinants of food waste. Attention will be given to spatial and temporal variations between different household types, individuals and habits. In the second phase, the mobile application will allow visibility of users' own as well as others' in home food availability and eco-feedback of wasted food. Results from this second phase are expected to give an indication of social interest as well as how visibility and eco-feedback affects behavior. Through the study of groups of interconnected users, we aim at the ability to see the potential for social solutions applied to food waste. This information could provide us with better indications on moments for susceptibility for persuasion and behavior change, which we eventually could use to inform the design of a social persuasive technology. In the third phase, a social recipe recommendation system for food ingredient sharing will be implemented. This concept is based on connecting users' in home food availability, which if combined, results in a social recipe aiming at collective food waste prevention. This type of communication technology is supported by Ganglbauers' [17] visibility dimension for cooperation as having potential to prevent food waste. They argued cooperation in multi-person households to be an essential ingredient to properly organize daily practices around food, e.g. to avoid duplicate buying or to share available food. From the user perspective the use of this system can help them to realize savings. The objective of this project is to contribute to an understanding of how the application of such a social media system can be integrated in consumers daily activities for efficient and effective but pleasurable food waste prevention.

\section{BACKGROUND}

\section{A. Factors influencing food intake and food waste behavior}

Food practices are influenced by a variety of factors. These factors can be biologically determined (such as taste and hunger) or depend on experience (such as associative conditioning and mere exposure). Furthermore, food practices can be influenced by intrapersonal factors (perceptions, norms, beliefs, attitude, knowledge etc.), interpersonal factors (social networks) and the environment (prices, resources, media, availability etc.) [18]. Knowing these factors is important as food choices can eventually result in detrimental effects on human health as well as on the environment. For example, people eat even when there is no hunger involved. When we smell food, dopamine is released to increase appetite [19]. This could result in difficulty to resist unnecessary shopping even after a meal or when food is available at home. Furthermore, in social settings where others are eating, we tend to comply and participate resulting in more food intake than usual. From a more fundamental perspective, even when we only observe or imagine someone eating a type of food we like, mirror neurons fire as if we perform the action ourselves [20], resulting in a higher probability of actually performing the action. These factors together with increasing demands on our time, everyday behaviors around food have become habitual or unconscious [20] [21]. This does not only affect human health (in the case of unhealthy food) but it could also result in food waste, which is an almost invisible result of previous decisions. Lack of planning, unpredictability of daily activities, overbuying, sellby dates or forgetting, were all found to contribute to food waste [21]. Even if intentions and attitudes are positive towards sustainable food behavior, it is not always reflected in everyday practice [22].

Pro-active behavior towards food waste, however, can prevent avoidable food waste in homes. This pro-active behavior can be triggered by economic reasons or because of growing up in a family with a lifestyle towards sustainability appreciating the food that is available [21]. Other factors predicting proenvironmental behavior are knowledge, being concerned about the environment together with certain personality traits (agreeable, openness) and gender [23] [24] [25] [26]. General beliefs, together with education and political affiliation, were further found to be stable predictors of overall environmental concern [27]. Evolutionary mechanisms also seem to prevent the adoption of sustainable food consumption resulting in occasional dosages of self-chosen ignorance. Griskevicius [28] proposed that a large portion of human-inflicted ecological damage is caused or exacerbated by five ancestral tendencies which have devastating consequences in the modern world; propensity for self-interest, motivation for relative status, proclivity to unconsciously copy others, predisposition to be shortsighted and proneness to disregard impalpable concerns. He suggests for influence strategies to be optimally effective, they must work with, rather than against, evolved tendencies. Another way to empower sustainability is to make sustainable actions appealing to the unconscious and impulse associative system. A sustainable action should be appealing and grabbing for rational reasons as well as gut-feeling [29].

\section{B. Current solutions for food waste prevention}

1) Public campaigns: Current influence strategies at the consumer level mainly aim at raising awareness in schools or through public campaigns [30]. The purpose of these strategies is to inform consumers about food purchase, storage, preparation, reuse of food residuals and actual shelf life of products. Due to a time span between the moment of being informed and the actual moments when a decision is made, this strategy could be ineffective. 
2) Human computer interaction: In [31] it was suggested that using the color code scheme in the refrigerator reduces the amount of expired food waste by increasing the awareness of available foods in the refrigerator for all members in a household. Clear et al. [32] evaluated the potential of design interventions for the practice of cooking. These include interactions with cooking appliances, digital meal sharing apps, group inventory management, and interactive technologies such as eco-feedback aiming at promoting awareness of and change towards alternative meals.

3) Social networks: A recent example is the BinCam [33], aiming at motivating reflection and behavioral change in the food waste and recycling habits of young adults. The system replaces an existing kitchen bin and automatically logs disposed items through digital images captured by a smart phone installed on the underside of the bin lid. Captured images are uploaded to a BinCam application on Facebook where they can be explored by all users of the system. Another example is Shareyourmeal, a platform allowing people to share their cooking with others in their neighborhood [34]. Overall though, little attention has been paid to the study of social influence strategies applied to food waste, while it has been found to have high potential as a means for generating positive behavior change [16].

4) Pervasive and mobile technology: In order to design technology to identify and influence food waste related practices, it is crucial to integrate technology with daily lives to catch the current habits truly as well as keeping the intervention with users at a minimum. Therefore, developments in pervasive technology applied to food waste behavior are getting increasingly important. The latest developments can be categorized in: (1) sensor based systems (RFID tagging or camera tracking), which are used to track the type and amount of waste, and (2) mobile applications to log and track food related activities.

In the field of smart home environments, food sensing technology is expected to have a significant impact on food waste prevention. Smart refrigerators can record expiration dates using bar code readers and RFID technology [35], so that food that is sensitive to spoiling can be closely monitored. The refrigerator may then alert when the food reaches a point where it must get used. Future research on smart refrigerators aims at an optimization of food identification with image and speech recognition techniques to improve the interaction process as well as to provide recipes based on recorded ingredients [35] [36]. RFID technology also plays an important role in pervasive technology research for waste identification. Here, a common technique to affect behavior is by increasing the visibility of waste flows [36] [37]. Furthermore, RFID is used to track the food in the supply chain to guarantee the quality of products more effectively [38]. The improvement of the process of food quality identification, is expected to reduce food waste in the cold supply chain. In addition to food sensing technology, an improved preservation technique is being developed that uses plastic circuits to understand actual food quality of packaged items to replace the currently misunderstood expiration date [39].

Mobile technology is another form of pervasive computing that can support food waste reduction. Adding the use of smart phones and cameras in the fridge improves the visibility of

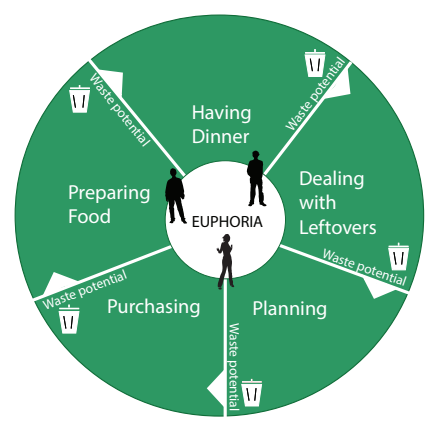

Fig. 1: Euphoria connects users throughout all food related behaviors.

in home availability [21]. In 2012, Ganglbauer et al. [17] presented a mobile food waste diary allowing consumers to reflect on how much food they actually waste. It addresses visibility in different phases of food practices for self reflection. The reasons of food waste were then made available to other users in order to encourage sharing and mutual reflection. Farr-wharton et al. [40] introduced a mobile application that can track the ingredients inside a color-coded refrigerator by taking automatic pictures and identifying foods. Their main goal was to alert users before the expiration date to achieve a reduction in food waste.

So far, still too little attention has been paid to food waste in households and preventing them through pervasive technologies. Food waste is a complex issue influenced by many factors throughout many food related practices. An ideal technological solution, should target the whole spectrum of food related behavior such as e.g. planning, purchase, preparation, cooking and disposal. With EUPHORIA we try to touch these behaviors through one system (Figure 1). Another potential for improvement is the provision of information on an intended behavior change instead of just focusing on behavioral feedback. According to Brynjarsdttir et al. [15] changing behavior towards sustainability is generally understood as reducing individual resource consumption without defining the intended behavior change. Systems mainly sense and measure the activity of resource consumption while the method of persuasion is restricted to just informing users. With EUPHORIA, we provide users with a solution by providing social recipes for food waste prevention.

\section{Methodology}

\section{A. Participants}

A longitudinal study will be carried out, initially targeting the Dutch population subdivided in students, professionals and $55+$ households in urban areas. Information about the study will be advertised through various social and professional networks.

\section{B. Apparatus}

A social mobile application is being developed for IOS and Android smart phones. The mobile application will be loadable from application stores of different mobile platforms. After download, users will be asked to enter information such as user names, age, occupation, area code etc. 


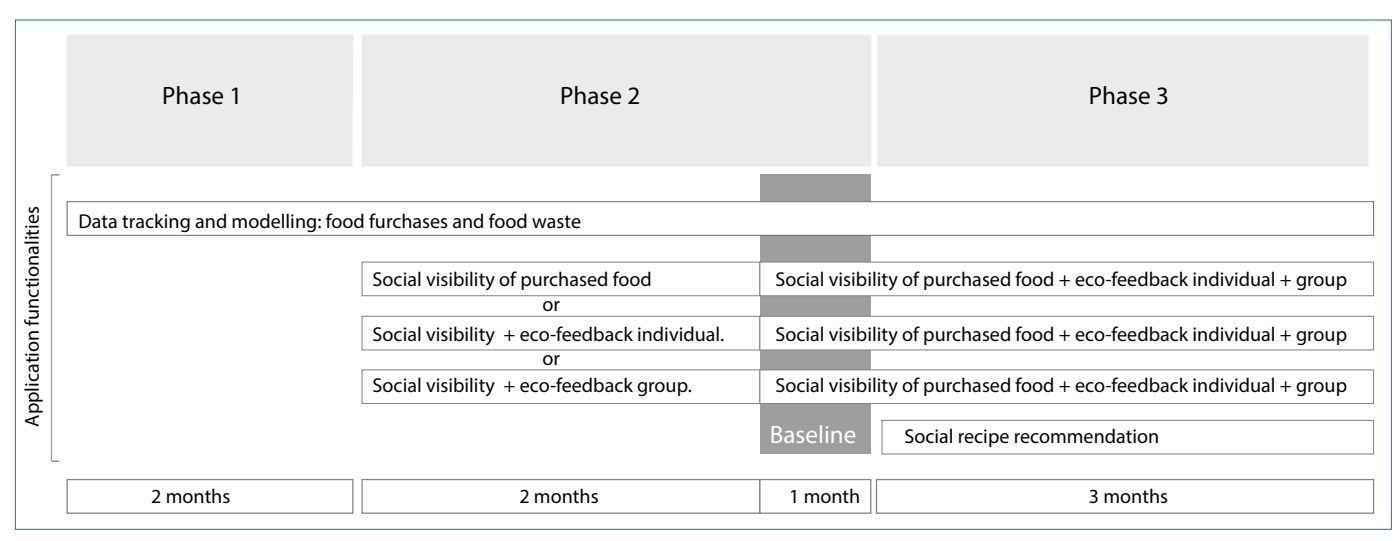

Fig. 2: Experimental setup.

1) Application functionalities: In the first version of the application (Phase 1), users can search for ingredients and select and enter its amount (in weight) to their shopping list or availability list. The shopping list is just a means to motivate data entering. For usability purposes, the application will provide a list of images (derived from image.net) of mostly used products to the user, allowing easy selection. Food that is consumed immediately in total (or planned to be consumed soon), do not need to be entered in the system. For entered data, users will be able to indicate which and how much food is used or wasted and the reasons of disposal and track this information anytime. Additionally, the system will provide a reminder that will be launched at least once a day to trigger users to enter data. In the second version of the application (Phase 2), new functionalities will be added. Users will be able to connect with friends, family or neighbors, disclose their food purchases and/or waste information, see each others' information and get eco-feedback of wasted food based on individual data and/or group data (if this data is disclosed by other users). Eco-feedback based on individual vs. group data could have different effects on different users [41]. The application will keep record of how social networks are formed and track whether users are interested in seeing other users' foods and waste activity, disclose theirs and use the ecofeedback function. In the third version of the application (Phase 3 ), users will be provided with recipe suggestions based on in home ingredients from connected users. The system will combine ingredients and come up with a recipe that first takes the oldest products while meeting the preferences of the whole group. In addition, the system will suggest right portions of food and provide recipes containing healthy alternatives and seasonal ingredients as much as possible. In this phase, we will track conformity to the recommendation and improve the system in such a way that is more likely for users to prevent food waste. See Figure 2 for the application functionalities in each phase.

2) Software design: The modules of the system and the data flow is demonstrated in Figure 3. The system works as a client-server model, with the mobile application as the client and a JAVA based server to respond to client requests and for serving data. The client side consists of a user interface and an activity tracker module that saves users' activities and sends them to the server. A database manager will keep track of the activities when internet is not available. The server application will have a database manager module consisting of user activities, food data and recipes. An online database will be used to retrieve the spatial and temporal data related to the dates of user activities. The neighbor manager module will be responsible for identifying a group of users that are most likely to conform to a social recipe recommendation. The Eco-Feedback Manager module will be responsible for giving feedback to people regarding their waste behavior. The social recipe recommender module will be used for finding the optimum recipe that is based on current ingredients of a group of users while satisfying the preferences of the group members. The data flow from the server to the client includes food information (ingredient names, ingredient types, the units of measurement, and images), recipe information and other users' food information and their activities. The flow from the client to the server consists of activities of the users including foods purchased, used and wasted and in which amount.

3) Implementation details: The application is being developed with an hybrid approach using PhoneGap in order to make it available for different mobile platforms. JQueryMobile, HTML and CSS are used for the user interface of the mobile application together with JavaScript for user interaction. The server side is being developed with PLAY framework for JAVA. The data flow between client and server is carried with JSON objects. Data from users will be stored in the local database of the smart phone by using an SQLite database engine and sent to the server database provided by PLAY framework (when internet is available).

\section{Procedure}

All participants will use all versions of the application developed throughout Phases 1, 2 and 3 for the same amount of time. Participants will also be asked to separate their food waste and dispose it in a bin with a weight sensor for data comparison. In addition, participants will be asked to collect receipts of purchases which will be used as cues for biweekly interviews to get information on the behavior around food use and waste to complement the data gathered through the mobile application as well as to test for its reliability. Objectives for every phase are discussed below. 


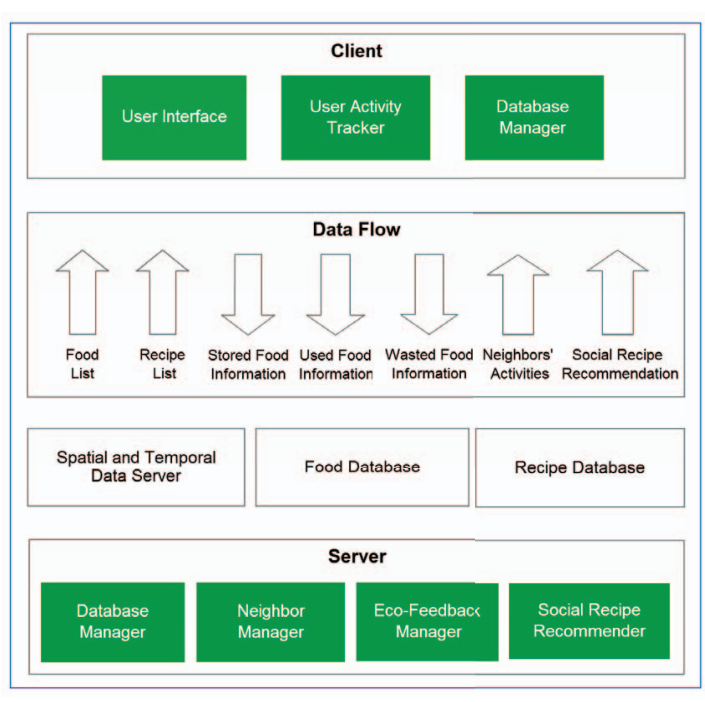

Fig. 3: System Framework

1) Phase 1: Monitoring and modeling food waste and food related behavior: In the first phase, the mobile application is implemented to monitor and model food related activities of users. The objectives of this phase is to determine the major activities on food practices and to understand key reasons of food waste. Entered data by users on food use and waste will be explored in how it is effected by type of day (e.g. workday vs. weekend), outside temperature and season. Usability tests will be done to ensure efficient interaction and satisfaction. To date, there has been little research in determinants of food waste behavior. Some qualitative data is available from Ganglbauer [17] [21], but quantitative data at a micro level is lacking. Most data on food waste are aggregated, lacking information on how and why consumers waste.

2) Phase 2: Eco-feedback and social visibility: Our final goal is food waste prevention through a group based recommendation system, a concept based on connecting users' in home food availability into one recipe. Therefore, it is very important to first explore people's attitude towards food information sharing and ingredient sharing. To measure the effect of social visibility and eco-feedback, participants will first be divided in groups in which each group receives different versions of the application; a version which only allows to see other users' available ingredients, a version allowing to see other users' available ingredients as well as eco-feedback of personal food waste behavior or a version allowing to see other users' available ingredients as well as eco-feedback of food waste behavior of their group (See Figure 2 for the experimental setup). Our assumption is that people are more likely to be persuaded by social recipe recommendations and social influences if they tend to share their information.

3) Phase 3: Introducing a social recipe recommendation system: With the concept of social recipes, we hope to touch multiple aspects that could result in food waste such as overbuying, forgetting and being social. While sharing food with other people may help overbuying problem, reminding users of their food may overcome forgetting it. Moreover, being social and willing to spend time with friends is given as another factor causing food waste [21]. Thus, we aim at using this behavior adversely so that people will spend their time with their friends by cooking their own food together that is balanced in nutritions while preventing it from getting spoiled.

\section{CONCLUSIONS, SOCIAL IMPLICATIONS AND FUTURE DIRECTIONS}

The aim of this project is to contribute to an understanding of how the application of a social media system can be integrated in consumers daily activities for efficient and effective but pleasurable food waste prevention. Although initially we will only use mobile applications to test out interactions, in the future, fully connected internet-of-things might support the home where appliances co-interact supporting sustainable lifestyles. The results from this project can be of great value for such future home appliances. EUPHORIA creates visibility at both household and community level and is expected to result in a more effective food waste prevention.

Our idea comes with social implications and challenges. As food practices are culturally integrated in personal lifestyles, the application of technology in the area of sustainable food practices can be a sensitive and complex topic to introduce in users' daily lives. We expect a need to stress the role of society in the trust and acceptance of technology. Social behavior around food practices is an important aspect of daily life and exploring it can have enormous impacts on the design, use and hence the acceptance of technology. Our main challenge lies in the collaboration with society which we will try to optimize through a participatory design process.

A related challenge is for users to gain trust in the technology which touches on privacy matters. Throughout the study we need to find the optimum balance between sustainable food practices and privacy. In the case of EUPHORIA, users have to manually input data and choose what information to share with others which allows them to control outgoing information, which we hope limits the issue of privacy. Influence strategies can then be based on available information. Therefore, users should trust, accept, have the perception of autonomy and be motivated to use the system for us to get a more complete insight into reasons of food waste and how to effectively prevent them. By suggesting users to use and share food that would otherwise get wasted, reduces unnecessary costs, which we hope to attract societies' interest. In economic terms, when consumers reduce their food purchases, money could be spend on other things which increases their welfare [13].

Another aspect worth mentioning is the health aspect. Our system will try to find and suggest optimal recipes based on available ingredients, users likes as well as food variety and healthy alternatives (for example reduced meat and diary consumption). Study have shown that in general, results will be greater if European households adopt healthy eating patterns in terms of lowering consumption of meats and dairy than if they only reduce food waste [13].

Finally, in addition to what is presented in this paper, a long term research goal is to look into the effects of the application mediated intervention on users' attitudes, perceptions, values, social connectedness etc. An interesting question here is whether food can be perceived or become a common good within a community, which requires a change in users' mindset. 


\section{ACKNOWLEDGMENT}

The authors would like to thank Lucio Marcenaro and Carlo Regazzoni from the Università degli Studi di Genova and Andreu Catala from the Universitat Politècnica de Catalunya for their feedback and suggestions.

\section{REFERENCES}

[1] J. Gustavsson, C. Cederberg, U. Sonesson, R. v. Otterdijk, and A. Meybeck, "Global food losses and food waste," Food and agriculture organization of the united nations, Tech. Rep., 2011.

[2] T. Fox Ceng, "Global food waste not , want not," The institution of Mechanical Engineers, Tech. Rep., 2013.

[3] "Almost half of the world's food thrown away, report finds," http://www.theguardian.com/environment/2013/jan/10/half-world-foodwaste, accessed: 2013-10-15.

[4] "Food waste facts," http://www.tristramstuart.co.uk/FoodWasteFacts.html, accessed: 2013-10-15.

[5] "Fusions," http://www.eu-fusions.org/, accessed: 2013-10-15.

[6] W. Moomaw, T. Griffin, K. Kurczak, and J. Lomax, "The critical role of global food consumption patterns in achieving sustainable food systems and food for all," United Nations Environment Programme, Tech. Rep., 2012.

[7] IPCC, "Climate Change 2007 : Synthesis Report," The Intergovernmental Panel on Climate Change., Tech. Rep. November, 2007.

[8] C. Beretta, F. Stoessel, U. Baier, and S. Hellweg, "Quantifying food losses and the potential for reduction in Switzerland." Waste management, vol. 33, no. 3, pp. 764-73, Mar. 2013.

[9] H. Soethoudt and T. Timmermans, "Monitor Voedselverspilling," Tech. Rep., 2013

[10] M. Kranert, G. Hafner, J. Barabosz, F. Schneider, S. Lebersorger, S. Scherhaufer, H. Schuller, and D. Leverenz, "Determination of discarded food and proposals for a minimization of food wastage in Germany - Abridged Version -," University Stuttgart. Institute for Sanitury Engineering, Water Quality and Solid Waste Management, Tech. Rep. February, 2012.

[11] T. Quested and H. Johnson, "Household Food and Drink Waste in the UK A report containing quantification of the amount and types of household," Tech. Rep. November, 2009.

[12] J. C. Buzby and J. Hyman, "Total and per capita value of food loss in the United States," Food Policy, vol. 37, no. 5, pp. 561-570, Oct. 2012.

[13] M. M. Rutten, "Reducing food waste by households and in retail in the eu: A prioritisation on the basis of economic, land use and food security impacts," in Proceedings of the First International Conference on Food Security, 2013, noordwijkerhout, Netherlands.

[14] _ "What economic theory tells us about the impacts of reducing food losses and/or waste: implications for research, policy and practice," Agriculture \& Food Security, vol. 2, no. 1, 2013. [Online]. Available: http://www.agricultureandfoodsecurity.com/content/2/1/13

[15] H. Brynjarsdóttir, M. Hå kansson, J. Pierce, E. P. S. Baumer, C. Disalvo, and P. Sengers, "Sustainably Unpersuaded : How Persuasion Narrows Our Vision of Sustainability," in CHI 2012, 2012, pp. 947-956.

[16] D. Foster and S. Lawson, "Liking Persuasion : Case studies in Social Media for Behaviour Change," in CHI 2013, 2013.

[17] E. Ganglbauer, G. Fitzpatrick, and G. Molzer, "Creating Visibility: Understanding the Design Space for Food Waste," in MUM 2012, 2012, pp. $0-9$.

[18] I. R. Contento, "Chapter 2 Overview of Determinants of Food Choice and Dietary Change : Implications for Nutrition Education," in Nutrition Education: Linking Research, Theory, and Practice. Jones \& Bartlett Publishers, 2010, pp. 26-42.

[19] A. Abizaid, Z.-W. Liu, Z. B. Andrews, M. Shanabrough, E. Borok, J. Elsworth, R. Roth, M. Sleeman, M. R. Picciotto, M. H. Tschöp, X.-B. Gao, and T. Horvath, "Ghrelin modulates the activity and synaptic input organization of midbrain dopamine neurons while promoting appetite," The journal of Clinical Investigation, vol. 116, no. 12, pp. 3229-3239, 2006.
[20] D. A. Cohen, "Neurophysiological pathways to obesity: below awareness and beyond individual control." Diabetes, vol. 57, no. 7, pp. 176873, Jul. 2008.

[21] E. Ganglbauer, G. Fitzpatrick, and R. Comber, "Negotiating Food Waste : Using a Practice Lens to Inform Design," ACM Transactions on Computer-Human Interaction, vol. 20, no. 2, pp. 1-25, 2013.

[22] I. Vermeir and W. Verbeke, "Sustainable food consumption among young adults in Belgium: Theory of planned behaviour and the role of confidence and values," Ecological Economics, vol. 64, no. 3, pp. 542-553, Jan. 2008.

[23] T. L. Milfont, J. Duckitt, and L. D. Cameron, "A Cross-Cultural Study of Environmental Motive Concerns and Their Implications for Proenvironmental Behavior," Environment and Behavior, vol. 38, no. 6, pp. 745-767, Nov. 2006.

[24] T. M. Laidley, "The Influence of Social Class and Cultural Variables on Environmental Behaviors: Municipal-Level Evidence From Massachusetts," Environment and Behavior, vol. 45, no. 2, pp. 170-197, Aug. 2011.

[25] M. G. Luchs and T. A. Mooradian, "Sex, Personality, and Sustainable Consumer Behaviour: Elucidating the Gender Effect," Journal of Consumer Policy, vol. 35, no. 1, pp. 127-144, Oct. 2011.

[26] C. Mobley, W. M. Vagias, and S. L. DeWard, "Exploring Additional Determinants of Environmentally Responsible Behavior: The Influence of Environmental Literature and Environmental Attitudes," Environment and Behavior, vol. 42, no. 4, pp. 420-447, Oct. 2009.

[27] A. Olofsson and S. Ohman, "General Beliefs and Environmental Concern: Transatlantic Comparisons," Environment and Behavior, vol. 38, no. 6, pp. 768-790, Nov. 2006.

[28] V. Griskevicius, S. M. Cantú, and M. v. Vugt, "The Evolutionary Bases for Sustainable Behavior: Implications for Marketing, Policy, and Social Entrepreneurship," Journal of Public Policy \& Marketing, vol. 31, no. 1, pp. 115-128, May 2012.

[29] C. Manning, "The Psychology of Sustainable Behavior," Ph.D. dissertation, 2009.

[30] R. Thönissen, "Fact Sheet : Food Waste in the Netherlands. Ministry of agriculture, Nature and Food quality," Tech. Rep. May, 2010.

[31] G. Farr-wharton, M. Foth, and J. H.-J. Choi, "Colour Coding the Fridge to Reduce Food Waste," in OZCHI 2012, 2012, pp. 119-122.

[32] A. Clear, M. Hazas, J. Morley, A. Friday, and O. Bates, "Domestic Food and Sustainable Design : A Study of University Student Cooking and its Impacts," in CHI 2013, 2013, pp. 2447-2456.

[33] A. Thieme, R. Comber, J. Miebach, J. Weeden, N. Krämer, S. Lawson, and P. Olivier, "We ve Bin Watching You Designing for Reflection and Social Persuasion to Promote Sustainable Lifestyles," in CHI 2012, 2012, pp. 2337-2346.

[34] "Share your meal," http://www.shareyourmeal.net/, accessed: 2013-1015 .

[35] J. Rouillard, "The Pervasive Fridge; A smart computer system against uneaten food loss," in The Seventh International Conference on Systems, no. c, 2012, pp. 135-140.

[36] L. Xie, Y. Yin, X. Lu, B. Sheng, and S. Lu, "iFridge: An Intelligent Fridge for Food Management based on RFID Technology," in UbiComp 2013, 2013, pp. 291-294.

[37] P. H. Krauchi, P. A. Wager, M. Eugster, G. Grossmann, and L. M. Hilty, "End-of-Life Impacts of Pervasive Computing : Are RFID Tags a Threat to Waste Management ?" IEEE Technology \& Society Magazine, Special Issue on Sustainable Pervasive Computing, vol. 24, no. 1, 2005.

[38] "Pasteur project," http://www.pasteur-project.info/, accessed: 2013-1015.

[39] "Plastic analogue-to-digital converter tests food freshness," http://www.electronicsnews.com.au/news/plastic-analog-to-digitalconverter-tests-food-fre, accessed: 2013-10-15.

[40] G. Farr-Wharton, M. Foth, and J. H.-J. Choi, "EatChaFood : Challenging Technology Design to Slice Food Waste Production," in UbiComp 2013, 2013, pp. 559-562.

[41] C. Midden and J. Ham, "Chapter 23 Persuasive technology to promote environmental behaviour," in Environmental Psychology; an introduction, 2013, pp. 243-254. 